\title{
Lipid Raft Microdomains of Bladder Epithelial Cells Regulates Apoptosis and Microenvironment Upon Exposure to C. albicans
}

Jayoung Kim ${ }^{1,2,3,4, ~}$, Austin Yeon ${ }^{1}$, Khandakar Tanvir Ahmed ${ }^{5}$, Wei Zhang ${ }^{5}$, Khae-Hawn Kim ${ }^{6}$, Woong-Ki Kim ${ }^{7}$

${ }^{1}$ Departments of Surgery and Biomedical Sciences, Cedars-Sinai Medical Center, Los Angeles, CA, USA; '2Samuel Oschin Comprehensive Cancer Institute, Cedars-Sinai Medical Center, Los Angeles, CA, USA; ${ }^{3}$ University of California Los Angeles, CA, USA; ${ }^{4}$ Department of Urology, Ga Cheon University College of Medicine, Incheon, South Korea; ${ }^{5}$ Department of Computer Science, University of Central Florida, Orlando, FL; ${ }^{6}$ Department of Urology, Chungnam National University Sejong Hospital, Sejong, Korea; ${ }^{7}$ Department of Microbiology and Molecular Cell Biology, Eastern Virginia Medical School, Norfolk, VA, USA

KEYWORDS. C. albicans; bladder; microarray; apoptosis; infection; microenvironment; lipid rafts; interstitial cystitis

\author{
${ }^{\top}$ Correspondence: \\ Jayoung Kim, PhD. \\ Departments of Surgery and Biomedical Sciences \\ Cedars-Sinai Medical Center, Davis 5071 \\ 8700 Beverly Blvd., Los Angeles, CA 90048 \\ Tel: +1-310-423-7168 \\ Fax: +1-310-967-3809 \\ E-mail: Jayoung.Kim@csmc.edu
}




\section{ABSTRACT}

INTRODUCTION. Interstitial cystitis/painful bladder syndrome (IC) is characterized by chronic bladder pain and urinary storage symptoms. IC affects more than 3.3 million women in the U.S. alone. Ibis T-5000 assays and next generation sequencing have revealed that the $C$. albicans fungus is highly abundant in the urine of IC patients, particularly those who report greater pain, urinary urgency, and flares. However, currently, the clinical significance of $C$. albicans in the urine remains elusive. Here, we report the pathological effects and mechanisms triggered by $C$. albicans in a healthy normal bladder.

METHODS. Immortalized bladder epithelial cells were infected with C. albicans. Perturbations in gene expression were identified using an Affymetrix gene microarray and subsequently followed with bioinformatic analyses, including gene set enrichment. Inflammatory and apoptotic genes were quantified using RT-PCR and Western blot analyses. Central signal pathways were examined using Western blot analysis.

RESULTS. DNA microarray analysis showed alterations in the transcriptome of bladder epithelial cells infected with $C$. albicans over both the short and long terms. Key inflammatory and apoptosis networks were changed, which was consistent with several cellular events. Cellular levels of reactive oxygen species and nitrogen oxide increased after infection. Productions of cyclooxygenase-2 and prostaglandine E2 also increased after C. albicans infection, and their productions were suppressed by blockage of reactive oxygen species-epidermal growth factor receptor-Erk pathway.

CONCLUSIONS. This study provides evidence that $C$. albicans infection triggers inflammation and cellular damage by dysregulating key regulatory genes, signaling pathways, and bioactive species in normal bladder cells. 


\section{ABBREVIATIONS}

IC

Interstitial cystitis/painful bladder syndrome

ROS

reactive oxygen species

EGFR

epidermal growth factor receptor

PLUS

The Prevention of Lower Urinary Tract Symptoms

LUTS lower urinary tract symptoms

UTIs $\quad$ urinary tract infections

OAB overactive bladder

UI

urinary incontinence

ITS

internal transcribed spacer

T2DM

Type 2 diabetes mellitus

AD

alzheimer's disease

$\mathrm{MCl}$

mild cognitive impairment

MAPP

Multidisciplinary Approach to the Study of Chronic Pelvic Pain

HRP

horseradish peroxidase

DEGs

differentially expressed genes

GO

Gene Ontology

KEGG

Kyoto Encyclopedia of Genes and Genomes

ECL

Enhanced Chemiluminescence Kit

MTT

3-(4,5-dimethylthiazol-2-yl)-2,5-diphenyltetrazolium bromide

TUNEL

Terminal deoxynucleotidyl transferase-mediated dUTP nick end labeling

ROS

Reactive Oxygen Species

NO

Nitric Oxide

DCFDA

2',7'-dichlorofluorescin diacetate

OCG

octylglucoside

BAD

BCL2 Associated Agonist of Cell Death 


$\begin{array}{ll}\text { BAX } & \text { BCL2 Associated } X \\ \text { TGFB1 } & \text { Transforming Growth Factor Beta-1 } \\ \text { NAC } & \text { N-acetyl-L-cisteine } \\ \text { COX-2 } & \text { cyclooxygenase-2 } \\ \text { PGE }_{2} & \text { prostaglandin E2 } \\ \text { GPI } & \text { glycosylphosphatidylinositol } \\ \text { MBCD } & \text { methyl- } \beta \text {-cyclodextrin } \\ \text { UCPPS } & \text { urologic chronic pelvic pain syndrome }\end{array}$




\section{BACKGROUND}

\section{Bladder Health in Women}

The Prevention of Lower Urinary Tract Symptoms (PLUS) Consortium recently defined the ideal female bladder health as, "a complete state of physical, mental, and social well-being related to bladder function and not merely the absence of lower urinary tract symptoms (LUTS). Healthy bladder function permits daily activities, adapts to short-term physical or environmental stressors, and allows optimal well-being (e.g., travel, exercise, social, occupational, or other activities)." LUTS among women include urinary tract infections (UTIs), overactive bladder (OAB), urinary incontinence (UI), interstitial cystitis/painful bladder syndrome (IC), etc. The Epidemiology of LUTS (EpiLUTS) study compiled the prevalence and associated effects of LUTS in the US, UK, and Sweden(1). This cross-sectional, population-representative survey found that the prevalence of LUTS is more than $75 \%$ in women older than 40 years old, and that most patients experienced a reduced quality of life. IC is a chronic bladder syndrome characterized by pain, pressure, and discomfort with urinary symptoms, such as urgency and frequency. The estimated yearly diagnosis of IC among women in the US is more than 3.3 million(2). The burdens of IC on public health and the lack of identified etiology remains a challenging issue in the field of urology.

\section{Role of Urinary Mycobiome in Health}

Humans have co-evolved with niche microorganisms that can be normally found in healthy individuals. Understanding the role of the mycobiome has become more and more important in human health and disease. Thanks to recently employed highly sensitive genomics tools and computational analyses, the mycobiome of the bladder has been explored. A series of studies analyzed the mycobiome of urine (voided or catharized) from healthy and diseased individuals. These recent findings, based on highly sensitive metagenomic technologies using fungal-specific internal transcribed spacer (ITS)-2 amplicon sequencing, revealed consistent patterns that show 
that the diversity of the mycobiome is reduced in diseased urine samples compared to healthy controls $(3,4)$.

A considerable amount of literature describes the important roles of the mycobiome in human health and disease. Fluctuations in fungal ecosystems may be linked to the abnormalities associated with diseases of the brain(5), heart, gastrointestinal track etc. Type 2 diabetes mellitus (T2DM) is associated dysbiosis in the gut mycobiome. Roles of the gut mycobiome in host immune system and homeostasis have also been suggested(6-9). Additionally, patients with alzheimer's disease $(A D)$ or mild cognitive impairment $(\mathrm{MCl})$ showed differences in their mycobiome compared to healthy individuals(10).

Although the relationship between the mycobiome and several diseases has been established, the contribution of the mycobiome in bladder homeostasis and alteration of urinary components is a relatively new perspective. Furthermore, the urinary mycobiome may be strongly associated with the bladder ecosystem. Perturbations of the mycobiome community can occur with pathological conditions or they may reflect underlying specific diseases(11-14). However, it remains uncertain whether specific mycobiome species are diagnostic or predictive of progression in urological diseases.

\section{IC, Mycobiome, and C. albicans}

While the etiology of IC remains unknown, much effort has been placed on identifying an infectious cause of IC; however, this has resulted in little to no success(15, 16). A previous study by the Multidisciplinary Approach to the Study of Chronic Pelvic Pain (MAPP) Research Network using the Ibis T-5000 Universal Biosensor System revealed that C. albicans was more abundant in urine samples obtained from IC patients who reported a flare (acute bladder pain), compared to those who did not(3). A follow-up study supported this finding and revealed elevated candidiasis 
[Odds Ratio $(\mathrm{OR})=6.63,95 \% \mathrm{Cl} 0.8-58.5, p=0.088)$ in IC patients who experienced more severe urinary symptoms, compared to those with little to no symptoms(4).

More than 200 Candida species have been found, and Candida albicans (C. albicans) is one of the most important fungal agents causing candiduria, followed by $C$. tropicalis (17-20). Although C. albicans is known as a benign mucosal fungal flora. it may lead disease when immunity is down. Candida species are globally emerging as pathogens of major public health concerns and are often found in UTIs. They cause a large spectrum of diseases, including vaginal and skin infections. Candida appears to widely affect people at least once in their lifetimes. Chronic exposure or use of antibiotics leads to a weakened immune system, allowing for easier infection with C. albicans. Infection is usually local, harmless, and easily treatable with antifungal drugs, such as fluconazole, flucytosine, and/or amphotericin B. Untreated infection can lead to systemic organ infection. A nosocomial candida infection afflicting the urinary tract, Candiduria can be defined as the presence of greater than $10^{5}$ fungal cfu/ml urine in adults, though as little as $10^{3} \mathrm{cfu} / \mathrm{ml}$ may also result in pathogenesis among groups at higher risk. When C. albicans is found in urine samples, it is related to the colonization of an indwelling catheter and/or the bladder, symptomatic cystitis, or invasive upper tract infection(21). C. albicans is the most frequently isolated species, but other species are increasingly gaining clinical significance.

The bladder wall has the well-defined layers including the mucosa which is the innermost portion of bladder wall. The mucosa is consisted of urothelium (the transitional epithelium that lines most structures of the urinary tract), basement membrane, lamina propria(22). Mucosal epithelial cells are the first line of barriers defending the invasion of $\mathrm{C}$. albicans. However, the interaction between $C$. albicans and bladder epithelial cells and the mechanism underlying urinary bladder infection caused by $C$. albicans are incompletely understood. 
Here, we aim to test the hypothesis whether infection with $C$. albicans causes bladder epithelial cell morphology alteration, cell damage, and production of inflammatory factors, and to characterize the mechanisms of pathogenesis in C. albicans infection of bladder epithelial cells, which may contribute to inflammation, cell apoptosis, and bladder-brain signaling. The findings from this study may further provide valuable information on the mechanisms underlying the infectious etiology related to IC. Elucidation of the potential strategies preventing the $C$. albicans infection will provide a greater understanding of $C$. albicans pathogenesis related to bladder health.

\section{MATERIALS AND METHODS}

\section{Cell lines and Cell Culture}

The immortalized human bladder epithelial cells, TRT-HU1, were maintained as described(23). The TRT-HU1 cell line was constructed and extensively characterized in previously published papers(23-28). The passage of cell lines was kept below 10, and mycoplasma contamination was tested for monthly via PCR analysis and confirmed negative before experiments. Cells were cultured in Dulbecco's modified Eagle's medium (DMEM), containing 10\% heat-inactivated fetal bovine serum [FBS, Invitrogen (Carlsbad, CA)], 1\% penicillin/streptomycin (Thermo Fisher Scientific), and $1 \%$ L-glutamine (Sigma-Aldrich Corp., St. Louis, MO, USA) in a $37^{\circ} \mathrm{C}$ humidified incubator with $5 \% \mathrm{CO}_{2}$. The culture medium was changed after one day of subculturing and cells were passed again when there was $70 \%-80 \%$ confluence.

For this study, C. albicans (Robin) Berkhout (MYA-2876 ${ }^{\mathrm{TM}}$, SC5314 (wild type)) were used (ATCC). After thawing the frozen stock of C. albicans, strains were cultured on yeast malt (YM) Agar (BD 271210) as instructed by company (BD, Franklin Lakes, NJ). They were picked from a 
single colony and grown in yeast malt medium containing amino acids plus $2 \%$ glucose at $30^{\circ} \mathrm{C}$. Strains were passaged twice in medium and harvested at the log growing stage. Cells were washed twice in phosphate-buffered saline (PBS) solution before cell counting on a hemacytometer.

To determine the biological effects of $C$. albicans infection in bladder epithelial cells, they were co-cultured with approximately $85 \%$ confluent TRT-HU1 $\left(1 \times 10^{6}\right.$ cells) with multiplicity of infection (MOI) $0.5,1,2,3$, or 4 for $2 \mathrm{~h}$. The co-culture plates were incubated in a $37^{\circ} \mathrm{C}$ humidified incubator with $5 \% \mathrm{CO}_{2}$.

\section{Antibodies and reagents}

The following specific antibodies were used for experiments: phospho-epidermal growth factor receptor (EGFR) (Y1068, active form), EGFR, phospho-Akt, Akt, phospho-p38, p38, phosphoErk, Erk, and $\beta$-actin from Cell Signaling Technology (Beverly, MA). Commercially available horseradish peroxidase (HRP)-conjugated secondary antibodies were obtained from Cell Signaling Technology. The remaining chemical reagents used for biological, biochemical, and function experiments were procured from Sigma-Aldrich (St. Louis, MO).

\section{Microarray analysis}

After co-incubation of TRT-HU1 cells and $C$. albicans at MOI 0.5 , the TRT-HU1 cells were harvested for sample preparation. First, reverse transcription of total RNA and subsequent steps for sample probe preparation, microarray hybridization, washing, and scanning of microarrays were performed following a standard Affymetrix Human Genome 430 Plus 2.0 Array (Affymetrix, Santa Clara, CA, USA) protocol at the UCLA Technology Center for Genomics \& Bioinformatics. The raw data was normalized using the gcrma package (version 2.10.0) in R 2.6.1. The log2 GCRMA signals were then exported and used for differential expression analysis. To identify 
differentially expressed genes (DEGs), a two-tailed Welch's t-test was implemented. DEGs were identified as genes with a $p$-value $<0.05$ and fold-change $\geq 1.5$. In order to reduce unreliable detection and false positives, probe sets with average expression levels higher than the average of all probe sets in the data were also considered for further analysis. The DAVID software was used to search for Gene Ontology (GO) terms and Kyoto Encyclopedia of Genes and Genomes (KEGG) pathways that were statistically enriched by the DEGs.

\section{SDS-PAGE gel running and Western blotting}

Cell lysates were prepared as described previously using a $4 \%$ sodium dodecyl sulfate-containing Iysis buffer. After quantification of protein concentration using Nanodrop, $25 \mu \mathrm{g}$ of proteins were mixed with a 4x protein sample loading buffer (10\% SDS, $500 \mathrm{mM}$ DTT, 50\% Glycerol, $500 \mathrm{mM}$ Tris-HCL and $0.05 \%$ bromophenol blue dye) (BioRad, Hercules, CA, USA). Protein running was performed using a $12 \%$ SDS-PAGE gel before transferring onto nitrocellulose membranes (BioRad, Hercules, CA, USA) for Western Blot analysis. The membranes were blocked with $5 \%$ skimmed milk at $37^{\circ} \mathrm{C}$ for 60 min and then immersed in primary antibodies $(1: 1000)$ at $4^{\circ} \mathrm{C}$ overnight. After washing with 1x TBST (0.1\% Tween-20, $10 \mathrm{mM}$ Tris-base and $100 \mathrm{mM} \mathrm{NaCl}$; pH 7) 3 times, the membranes were incubated for $1 \mathrm{~h}$ with a HRP-conjugated secondary antibody (1:2000). After additional TBST washes (3 times and $10 \mathrm{~min}$ each), an Enhanced Chemiluminescence Kit (ECL; Thermo Fisher Scientific, MA, USA) was applied. The Gel \& Blot Imaging System was used for visualization of the protein bands and quantification of band intensity was done by using ImageJ.

\section{Cell assay}

To test cell growth after exposure to $C$. albicans, TRT-HU1 cells were seeded onto $10 \mathrm{~cm}$ plates with a density of $5 \times 10^{6}$ cells/plate. Cells were maintained in standard growth medium for 1 day and then incubated with varying doses of $C$. albicans for 24,48 , or $72 \mathrm{~h}$. Cell proliferation was 
measured by manually counting cells using a hemocytometer. The averages of each count were used as the total density of the well after each time point.

After incubating with media containing C. albicans for the indicated times, cells were fixed with $4 \%$ paraformaldehyde at room temperature for $5 \mathrm{~min}$. For crystal violet staining, cells were stained with $0.05 \%$ crystal violet for $15 \mathrm{~min}$. Tap water was used to wash any extra staining. The cells were dried on filter paper, and the plates were scanned and quantified as described(29). For quantitative analysis, a $10 \%$ acetic acid solution was used to dissolve the stained cells, and absorbance at 570-590 nm was measured. All experiments were run in triplicates, and the data are representative of three independent trials.

Cell viability was determined by uptake of 3-(4,5-dimethylthiazol-2-yl)-2,5-diphenyltetrazolium bromide (MTT). To measure cell viability, MTT solution was added to the culture medium, according to the manufacturer's instructions (Abcam, Cambridge, UK). After staining, absorbance was measured at a wavelength of $570 \mathrm{~nm}$ on a microplate reader. All data are representative of 6 independent trials. All other reagents were obtained from Sigma-Aldrich or Promega (Madison, WI).

To detect DNA fragmentation related to apoptosis, a TUNEL assay was performed using the TUNEL Assay Kit - FITC (Abcam) after fixating cells with 4\% formaldehyde for 15 min on ice. Fragmented DNA was labeled with fluorescein using the TUNEL reaction mix and then analyzed with flow cytometer or fluorescence microscope(30).

\section{ELISA apoptosis analysis}


An ELISA for quantitative apoptosis was used to measure the levels of apoptosis according to the protocol provided by company (Cell Death Detection ELISA; Roche Diagnostics Corp.).

\section{Terminal deoxynucleotidyl transferase-mediated dUTP nick end labeling (TUNEL) assay} Apoptotic cells were also quantified by nuclear fragmentation detection by TUNEL (green) by counting the TUNEL-positive bladder epithelial cells under a microscope.

\section{Measurement of Reactive Oxygen Species (ROS) and Nitric Oxide (NO)}

A 2',7'-dichlorofluorescin diacetate (DCFDA) assay to quantitatively assess ROS was performed by using the Intracellular ROS Detection Assay Kit (Abcam), as described in literature(31). Cells were treated with $C$. albicans under the indicated conditions prior to the assay. Fluorescence spectroscopy with excitation/emission at $490 / 570 \mathrm{~nm}$ was used to detect fluorescence and measure the levels of ROS in response to infection. The control condition (without C. albicans) was equated $100 \%$ with the treatment conditions proportionally. Signals were background corrected and adjusted to cell numbers. For NO detection, a commercial NO Assay Kit (colorimetric, Abcam) was used, and total nitrate/nitrite amounts were measured in a simple twostep process(32).

\section{Lipid raft isolation}

Lipid raft membranes were isolated using differential extraction with Triton $\mathrm{X}-100$ and octylglucoside (OCG) detergent. Triton-soluble and insoluble (OCG-soluble) membrane components were isolated, as described in previous literature(33). All experimental steps were done on ice or at $4^{\circ} \mathrm{C}$.

\section{Statistical analysis}


All experiments were repeated at least three times, in most cases six times, with independent biological triplicates. Each of the experiments did not show significantly different results among repetitions. Data was analyzed using an unpaired two-tailed Student's t-test or one-way ANOVA using Prism (GraphPad Software, Inc., La Jolla, CA). Mean values from 6 biological replicates were used for statistical analyses, and all data were presented as the mean \pm standard deviation (SD). Students' t test and one-way ANOVA post-hoc Tukey's test were used to compare groups of data. Statistical significance for all analyses was determined at $p<0.05$.

\section{RESULTS}

\section{Apoptotic signatures induced by $C$. albicans infection}

In this study, we sought to investigate the mechanism underlying alterations in the human bladder in response to $\mathrm{C}$. albicans. We first found that infection with $C$. albicans led to morphological alterations and cellular damage in TRT-HU1 bladder epithelial cells. Co-culture under the conditions of MOI 2 or 3 induced the dramatic morphological changes of TRT-HU1. Under MOI 2 and $\mathrm{MOI} 3$, most of cells were started altering to a rounded morphology after $3 \mathrm{~h}$ co-culture. After $12 \mathrm{~h}$ co-culture, microscopic observation showed that approximately over $70 \%$ of the cells were detached from culture plate both at MOI 2 and MOI 3 (Fig 1A). Because the co-culture condition at MOI 0.5 for $3 \mathrm{~h}$ did not show any morphological alterations nor reduced cell viability, we decided to use this condition for further molecular study.

To further investigate the cytotoxic effects of $C$. albicans on bladder epithelial cells, cell mass was measured using crystal violet staining (Fig 1B). Apoptosis analyses were performed using tryptophan staining and MTT assay (Fig 1C, D). TUNEL staining also supported the increased apoptosis of bladder epithelial cells infected with C. albicans (Fig 1E). An apoptotic biomarker, 
cleaved PARP, was detected from Western blot analysis (Fig 1F). C-PARP gradually increased in response to $C$. albicans infection, while protein expression of cyclin D1 was suppressed. This decrease was accompanied by an increase in levels of PARP cleavage form.

\section{DNA microarray analysis shows induced apoptotic signatures after $C$. albicans infection}

To gather molecular evidence of these observations, DNA microarray analyses were performed, which revealed that the global gene expression of normal human bladder epithelial cells were significantly changed after infection with C. albicans.

In total, 5,213 genes were found to be differentially expressed after $C$. albicans exposure, compared to controls. A heatmap shows 5,213 DEGs, containing 1,144 upregulated and 4,069 downregulated genes 30 min after $C$. albicans exposure (Fig 2A). A similar analysis identified 1,044 DEGs with 606 upregulated and 438 downregulated genes $3 \mathrm{~h}$ after $\mathrm{C}$. albicans exposure (Fig 2B). A z-score transformation was applied to the original gene expression values when generating the heatmaps. There were 547 overlapping DEGs between the two gene lists (Fig 2C). Supplementary Figures $\mathbf{A}$ and $B$ shows the quality assessment. The numbers of overlapping upregulated and downregulated genes are shown in Figs S2A and S2B. The top 20 DEGs 30-min and 3-h after C. albicans exposure are shown in Fig 2D and 2E, respectively.

Gene enrichment analyses suggested that genes indicative of apoptosis greatly enriched $3 \mathrm{~h}$ after C. albicans infection (Fig 2E). Expression levels of apoptotic proteins were also analyzed. Genes associated with regulation of apoptosis, such as BCL2 Associated Agonist of Cell Death (BAD), BCL2 Associated X, Apoptosis Regulator (BAX), and Transforming Growth Factor Beta-1 (TGFB1) were upregulated in response to C. albicans infection (Fig 2E). Collectively, our results show that $C$. albicans infection induces transcriptomic alterations, leading to eventual apoptosis in bladder epithelial cells. 


\section{Induced apoptosis of human bladder epithelial cells via ROS and NO production by $C$.}

albicans infection

Activation of apoptosis includes ROS production, leading to damage of proteins, nucleic acids, lipids, etc. To determine whether $C$. albicans infection causes ROS and other reactive species, intracellular levels of ROS were measured at different time points (Fig 3A, left). The results were further validated when treatment with antioxidant $\mathrm{N}$-acetyl-L-cisteine (NAC) suppressed ROS production to almost baseline (Fig 3A, right). Western Blot analysis of c-PARP showed upregulation of ROS in response to $C$. albicans infection was almost completely diminished by NAC pretreatment (Fig 3B). In contrast to ROS, there was a decrease in NO production with $C$. albicans infection (Fig 3C). In consistent with this finding, Western blot analyses indicated decreased NOS protein (eNOS and iNOS) production in response to C. albicans (Fig 3D).

Acute infection of $C$. albicans activates inflammatory networks via activation of EGFR signaling pathways

In the following experiments, we examined the inflammatory responses, including cyclooxygenase-2 (COX-2) protein expression and prostaglandin E2 (PGE $)$ secretion into conditioned media (Fig 4A-B). In addition, the secretion levels of $\mathrm{PGE}_{2}$ were greatly inhibited when ROS was diminished by NAC treatment (Fig 4B).

Given that ROS production stimulates the activation of key signaling molecules, a series of signaling pathways were tested. Among MAPK pathways, phosphorylation of ERK, p38, and JNK each were determined. Western Blot analysis suggested the temporary deactivation of EGFR and Erk MAPK following C. albicans exposure for $15 \mathrm{~min}$. In contrast, the p38 MAPK was activated, as indicated by increased phosphorylated form of p38 (Fig 4C), while EGFR and Erk MAPK were deactivated. However, these were temporary events that recovered soon after time passed. 
These findings are aligned with the previous literature showing that induction of COX-2 gene by Candida albicans through EGFR, ERK, and p38 pathways in human urinary epithelium(34).

Because ROS stimulated the activation of EGFR signaling pathway, we investigated whether EGFR inhibition can suppress $\mathrm{PGE}_{2}$ secretion, and if NAC can have further effects. Although modest, NAC showed additional inhibition of EGFR inhibitor on extracellular $\mathrm{PGE}_{2}$ secretion (Fig 4D). To further understand the role of the secreted $\mathrm{PGE}_{2}$ in bladder microenvironments, the conditioned medium was collected from the C. albicans-infected bladder cells. After removal of cell debris, RAW264.7, a mouse monocyte/macrophage-like cell line, was challenged by incubation in the collected conditioned medium. We found a significant stimulation of proliferation in Raw 264.7 cells incubated in the conditioned medium (Fig 4E). As a control, the conditioned medium collected in absence of $C$. albicans was used.

\section{Lipid rafts microdomains are essential for C. albicans to stimulate the ROS-EGFR signaling pathway}

Lipid rafts are dynamic plasma membrane microdomains. Being enriched with cholesterol, glycosphingolipids, glycosylphosphatidylinositol (GPI)-linked proteins, lipid rafts play key roles as platforms for diverse signaling molecules. Key cellular receptors are co-localized and regulated, contributing to the transduction of signaling cascades. Disruption of lipid rafts by methyl- $\beta$ cyclodextrin $(M \beta C D)$ blocks the signaling networking. Thus, lipid rafts may be important in $C$. albicans-induced cell damage on bladder epithelial cells. We tested this hypothesis and found that $M \beta C D$ suppresses $C$. albicans-induced ROS production and apoptosis (Fig $5 \mathbf{A}$ ). Cell fractionation and enrichment showed that lipid raft microdomains contained EGFR (Fig 5B, top panel). 
Upon C. albicans infection, a portion of EGFR (approximately $50 \%$ ) translocated into the non-lipid rafts domains. As a result, the EGFR found on lipid rafts decreased with $C$. albicans infection (Fig 5B, second panel). A-Tubulin and Gia2 were used as marker proteins of non-raft and raft domains, respectively (Fig $5 \mathrm{~B}, 3^{\text {rd }}$ and $4^{\text {th }}$ panels). Furthermore, we addressed whether lipid raft disruption by cholesterol-interfering treatments affected EGFR translocation mediated by $C$. albicans infection. The decreased levels of EGFR on lipid rafts were recovered by an inhibition of lipid rafts and/or ROS production (Fig 5C). Collectively, these findings suggest that lipid rafts participate in the process of $C$. albicans infection in bladder epithelial cells.

\section{DISCUSSION}

Our present demonstrated what molecular events occur when the bladder is infected with $C$. albicans. We aimed at analyzing the role of $C$. albicans in the bladder because mycobiota communities exist in urine, and the interactions between these communities and the microenvironment are likely to be critical for normal bladder health.

Our hypothesis was inspired by previous studies by colleagues that reported fungal communities from voided urine samples collected from female and male patients with chronic lower urinary track symptoms and healthy controls(4, 35-37). These findings were based on a cultureindependent next-generation sequencing approach combined with computational analysis. Their data suggested that $C$. albicans is the most abundant fungal species detected in voided urine specimens of urologic chronic pelvic pain syndrome (UCPPS) patients compared to healthy controls. A series of reports have demonstrated that a large complex fungal community exists in urine. The extent of mycobiota and human interactions in the urinary tract may be linked to their clinical significance; however, such theories are extremely rough for now. Little is known about 
the human urine mycobiomes in disease states, even though current evidence points toward a potential central role in bladder dysfunction. This could be attributed to the fact that relatively few fungal genomes have been sequenced and annotated.

The experimental data from this study demonstrated that lipid raft microdomains participate in orchestrating the relocation of EGFR, a protein kinase receptor, which is a key regulator of defense mechanisms against $C$. albicans infection-induced cell damage. Lipid rafts microdomains were found to participate in the infection process of a few other pathogens. When disrupting lipid rafts through treatment with $M \beta C D$, a cholesterol-extracting agent, human monocytes were not able to uptake $C$. albicans (38). While more data are still needed, these findings suggest that $C$. albicans infection could induce cell damage through signaling activation and lipid raft microdomains, as illustrated in Fig 5D. These results also pave the way for future studies to decipher the niche and relationship between the bladder, its microenvironments, and the local mycobiome. Furthermore, these findings also suggest the possible therapeutic strategy of lipid raft disruption on $C$. albicans-induced immune response and pathological damage on bladder epithelial cells.

\section{STATEMENTS AND DECLARATIONS}

\section{ETHICS APPROVAL AND CONSENT TO PARTICIPATE}

N/A

\section{CONSENT TO PUBLISH}

The authors consent to publish. 


\section{AVAILABILITY OF DATA AND MATERIALS}

The datasets used during the current study are available from the corresponding author on reasonable request.

\section{COMPETING INTEREST}

The authors have nothing to disclose.

\section{FUNDING}

This research was funded by National Institutes of Health (1U01DK103260, 1R01DK100974, U24 DK097154, NIH NCATS UCLA CTSI UL1TR000124), Department of Defense (W81XWH-15-10415 and W81XWH-19-1-0109), Centers for Disease Controls and Prevention (1U01DP006079), and the U.S.-Egypt Science and Technology Development Fund by the National Academies of Sciences, Engineering, and Medicine (all to J.K.). This article is derived from the Subject Data funded in whole or part by National Academies of Sciences, Engineering, and Medicine (NAS) and The United States Agency for International Development (USAID). Any opinions, findings, conclusions, or recommendations expressed in this article are those of the authors alone, and do not necessarily reflect the views of USAID or NAS.

\section{AUTHOR CONTRIBUTIONS}

$\mathrm{JK}$ and $\mathrm{AY}$ drafted main manuscript, $\mathrm{WK}$, and $\mathrm{KK}$ wrote and revised the manuscript text, JK, AY, and KK prepared and analyzed data, and KTA and WZ prepared figures 1, S1 and S2. All authors reviewed the manuscript.

\section{ACKNOWLEDMENTS}


This research was supported by the Samuel Oschin Comprehensive Cancer Institute (SOCCI) at Cedars-Sinai Medical Center through 2019 Lucy S. Gonda Award. We appreciate technical support from the Cedars-Sinai Proteomics and Metabolomics Core. 


\section{FIGURE LEGENDS}

Figure 1. Cell damage and apoptosis were induced by $C$. albicans infection. (A) TRT-HU1 bladder epithelial cells were detached from culture plate after co-culture with C. albicans. Coculture of cells with $C$. albicans for $0,3,6,9$, and $12 \mathrm{~h}$ under $\mathrm{MOI}=0,0.5,1,2$, or 3 . Medium only $(\mathrm{MOI}=0)$ served as the control. (B) Cell mass was quantified by crystal violet staining assay. (CD) Time dependent apoptosis and viability in response to C. albicans infection. (C) Viability of cells was measured by MTT assay. (D) Apoptosis rates were quantified by trypan blue staining assay. (E) Cells under apoptosis were shown by TUNEL staining assay (green). (F) Protein expression levels of c-PARP and cyclin D1 were measured by Western blot analysis following 3, 12,24 , and $36 \mathrm{~h}$ of co-culturing with $\mathrm{C}$. albicans under $\mathrm{MOI}$ 0.5. As the internal loading control, $\beta$ actin was used.

Figure 2. DNA microarray analysis of apoptotic signatures induced by C. albicans. The differentially expressed genes (DEGs) by $C$. albicans infection in bladder epithelial cells were identified. (A) Heatmap showing the DEGs between control and 30 min after C. albicans coculture. (B) Heatmap showing the DEGs between control and $3 \mathrm{~h}$ after $\mathrm{C}$. albicans co-culture. (C) Venn diagram shows the overlapped DEGs from the two lists. (D-E) Up or downregulated cellular

processes of DEGs (D, 30 min; E, $3 \mathrm{~h}$ after $C$. albicans co-culture). Over-representation analysis showing pathways that are up or downregulated in bladder epithelial cells infected with $C$. albicans vs. controls at the indicated time points.

Figure 3. Apoptosis was induced by C. albicans infection via ROS and NO production. (A) The time-dependent ROS production in response to $C$. albicans infection (left). The $C$. albicansinduced ROS production was suppressed by NAS, a ROS inhibitor (right). (B) Western blot analysis showed that the C. albicans-induced cell apoptosis is diminished by NAS treatment. (C) 
NO production was quantified as described in the Materials and Methods. (D) Western blot analysis of eNOS and iNOS protein expression. The results were derived from six independent assays and presented as the mean $\pm \mathrm{SD}\left({ }^{*}, P<0.05\right)$.

Figure 4. Acute infection of $C$. albicans activated inflammatory networks via EGFR signaling pathway. (A) Production of COX-2 after C. albicans infection. (B) Production of PGE 2 after $C$. albicans infection. Treatment with NAC suppressed $\mathrm{PGE}_{2}$ production stimulated by $C$. albicans infection. (C) Activation of EGFR in bladder epithelial cells was induced by $C$. albicans infection. Protein levels of phosphorylated Erk and p38 MAPK were analyzed using Western blot analysis. $\beta$-actin served as the loading control. (D) Blockage of EGFR signaling suppressed stimulation by $C$. albicans infection. (E) Inflammatory responses to $C$. albicans. Proliferation of RAW 264.7 macrophage cells were stimulated by the conditioned medium secreted from C. albicans-infected bladder epithelial cells. The results were derived from six independent assays and presented as the mean $\pm \operatorname{SD~}\left({ }^{*}, P<0.05\right)$.

Figure 5. Lipid rafts are essential for C. albicans-induced signaling pathways and ROS production. (A) ROS production and apoptosis levels were increased by C. albicans infection. When lipid raft microdomains were disrupted by $M \beta C D$ treatment, ROS production and apoptosis levels decreased. The results were derived from three independent assays and presented as the mean $\pm \mathrm{SD}(*, P<0.05)$. (B) EGFR is in lipid raft microdomains, while EGFR translocated into non-raft membranes in response to $C$. albicans infection. (C) The lipid raft localization of EGFR is altered in response to $C$. albicans infection. Both NAS and M $\beta C D$ blocked the localization of EGFR on the lipid raft microdomains. (D) Schematic illustrating pathways that are potentially involved in biological events in human urothelial cells and their microenvironments. 
Figure 1.

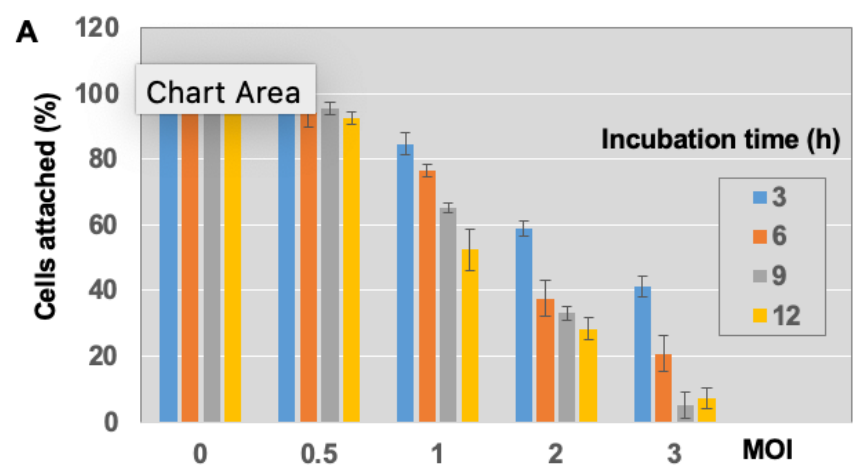

B
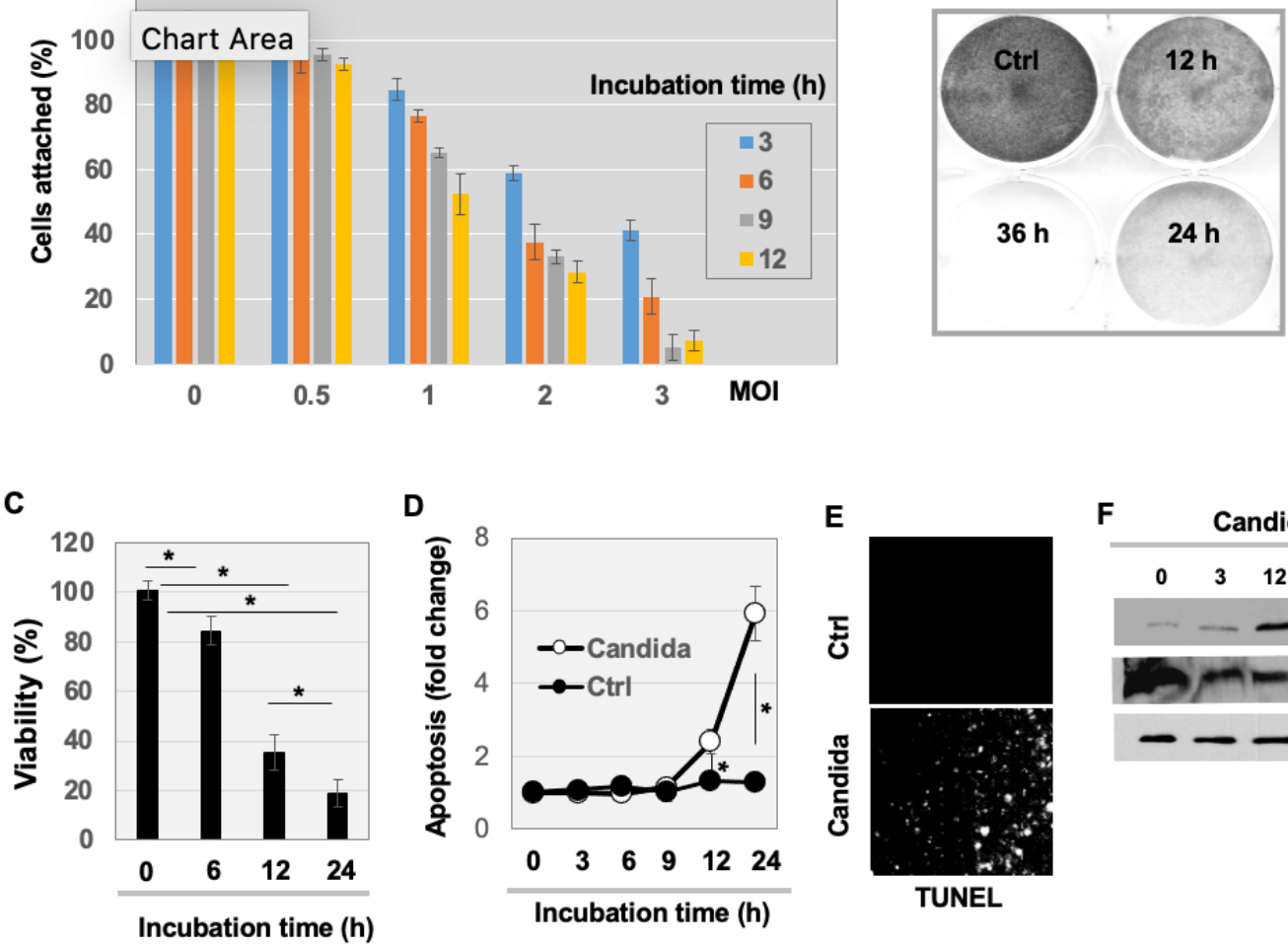

D

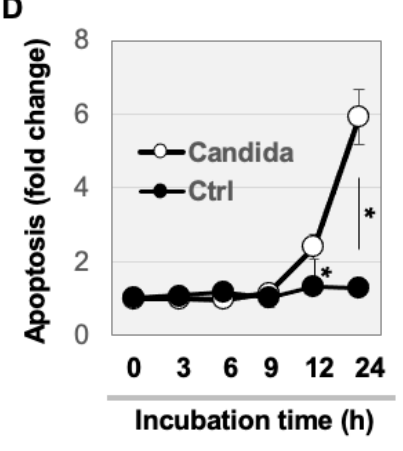

E

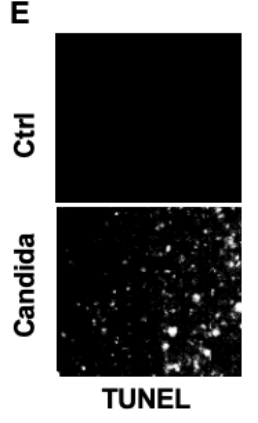

$\mathbf{F}$

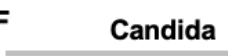

$\begin{array}{llllll}0 & 3 & 12 & 24 & 36 & \text { (h) }\end{array}$

- - - C-PARP

Cyclin D1

$--\infty-\beta$ Actin 
A

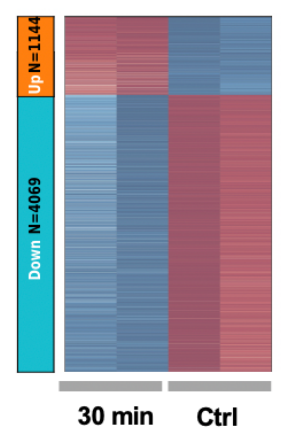

C

Differentially expressed genes

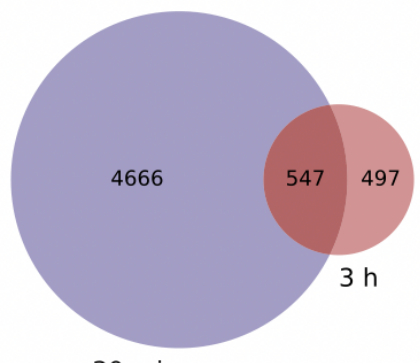

$30 \mathrm{~min}$
B

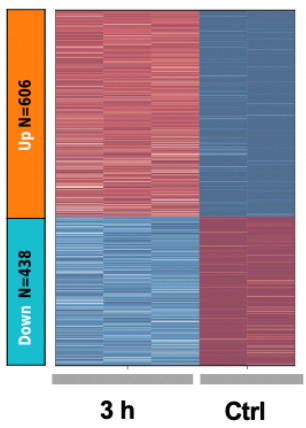

E
D

\begin{tabular}{|cccc|}
\hline \multicolumn{5}{c}{ Up or Down-regulated Cellular Processes (30 $\mathbf{m i n})$} \\
\hline Category & Term & Count & P value \\
\hline Up & Skeletal system morphogenesis & 14 & 0.002681 \\
Up & Microtubule-based process & 22 & 0.010168 \\
Up & Cell projection organization & 29 & 0.010567 \\
Up & Neuron development & 26 & 0.021283 \\
Up & Mesenchyme development & 7 & 0.037067 \\
Up & Chromatin modification & 21 & 0.040597 \\
Up & Regulation of protein stability & 6 & 0.048998 \\
\hline Down & RNA processing & 235 & $8.63 \mathrm{E}-28$ \\
Down & Cell cycle & 286 & $3.08 \mathrm{E}-20$ \\
Down & mRNA metabolic process & 160 & $1.86 \mathrm{E}-19$ \\
Down & Mitotic cell cycle & 151 & $3.75 \mathrm{E}-15$ \\
Down & Golgi vesicle transport & 63 & $2.27 \mathrm{E}-10$ \\
Down & Oxidative phosphorylation & 50 & $1.70 \mathrm{E}-09$ \\
Down & Amine biosynthetic process & 31 & 0.001921 \\
\hline
\end{tabular}

\begin{tabular}{|c|c|c|c|c|}
\hline Category & Term & Count & Pvalue & DEGs belong to the \\
\hline Up & Regulation of apoptosis & 55 & $1.02 E-06$ & Regulation of Apoptosis \\
\hline Up & Regulation of cell death & 55 & $1.56 \mathrm{E}-06$ & \\
\hline up & Golgi vesicle transport & 18 & $2.01 E-06$ & $\begin{array}{l}\text { ABL1, ACTN4, BAD, BAX, } \\
\text { BNIP1, BOK, BRCA2 }\end{array}$ \\
\hline Up & Protein transport & 49 & $2.31 \mathrm{E}-05$ & BTG1, CD70, CSF2, DAP, \\
\hline Up & Anti-apoptosis & 20 & $7.466-05$ & DAPK3, DNM2, EGFR, \\
\hline Up & Vesicle coating & 6 & $9.866-05$ & EIF5A, ERCC2, F2R, \\
\hline Up & Positive regulation of apoptosis & 29 & $7.33 E-04$ & FOXC1, IFI6, PDIA3, \\
\hline Up & Regulation of JAK-STAT cascade & 7 & 0.001197 & HMGB1, HMOX1, HSPA5, \\
\hline Up & Vesicle-mediated transport & 34 & 0.002225 & IL1A, TNFRSF9, \\
\hline Up & Cell cycle process & 31 & 0.009857 & $\begin{array}{l}\text { ILK, MSX2, P2RX4, } \\
\text { SERPINB2 PHB, PPP2CA }\end{array}$ \\
\hline Up & Positive regulation of cell migration & 9 & 0.010465 & PPP2R1A, PRKCE, SOD2, \\
\hline Up & Regulation of cell size & 15 & 0.010550 & TBX3, TERT, TGFB 1 , \\
\hline Up & Regulation of calcium ion transport & 7 & 0.029044 & THBS1, TNFAIP3, VDR, \\
\hline Up & Small GTPase mediated signal transduction & 18 & 0.030703 & VHL, CUL1, AIFM1, \\
\hline up & Glycoprotein metabolic process & 13 & 0.042245 & BCLAF1, DNAJB6, ATF5, \\
\hline Down & Regulation of transcription & 87 & $2.37 \mathrm{E}-08$ & $\begin{array}{l}\text { PHLDA3, PRDX5, AATF, } \\
\text { APH1A SCAND1 TAFG }\end{array}$ \\
\hline Down & Chromatin modification & 13 & 0.005877 & PSENEN, MKL1, AKT1S1 \\
\hline Down & Cellular protein catabolic process & 19 & 0.036691 & \\
\hline
\end{tabular}


Figure 3.

A

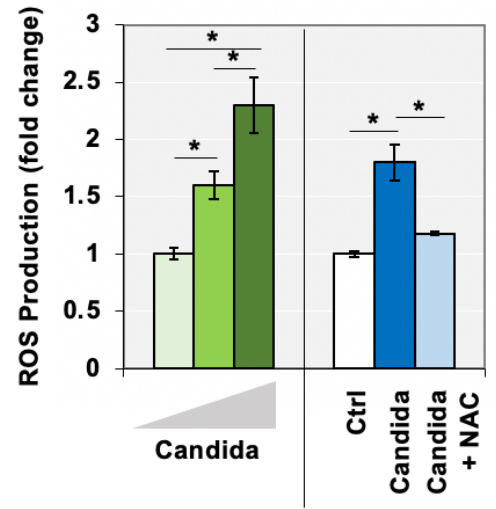

C

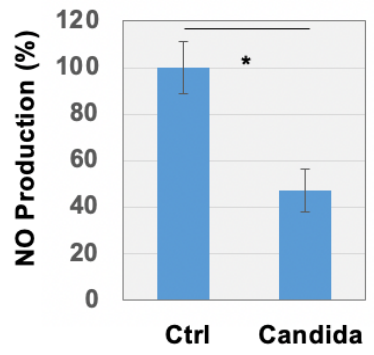

B

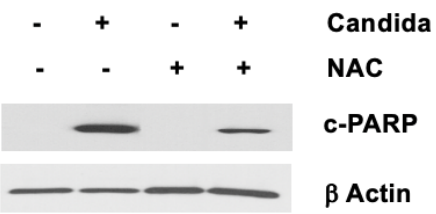

D

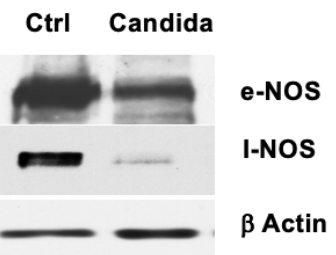




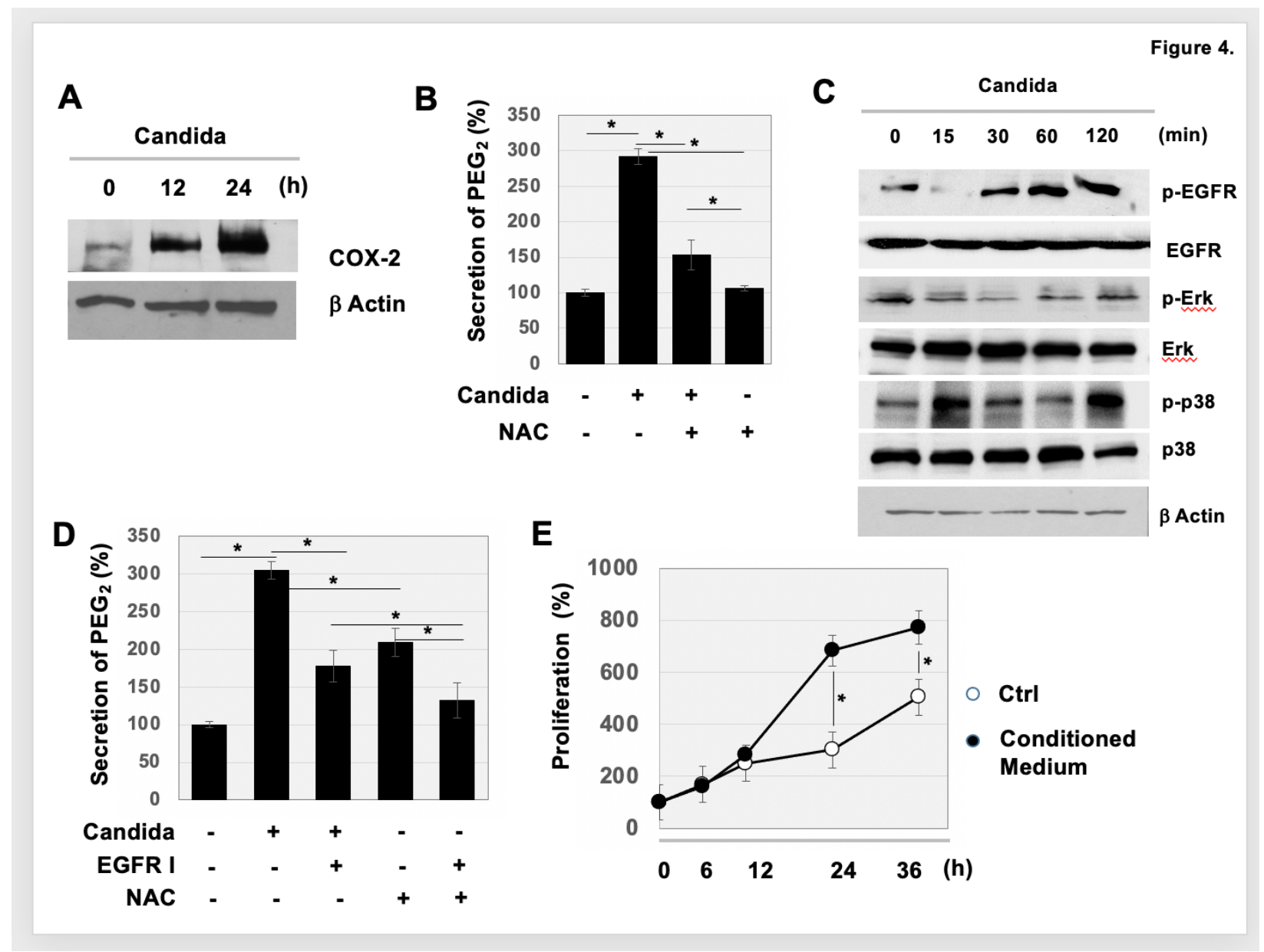




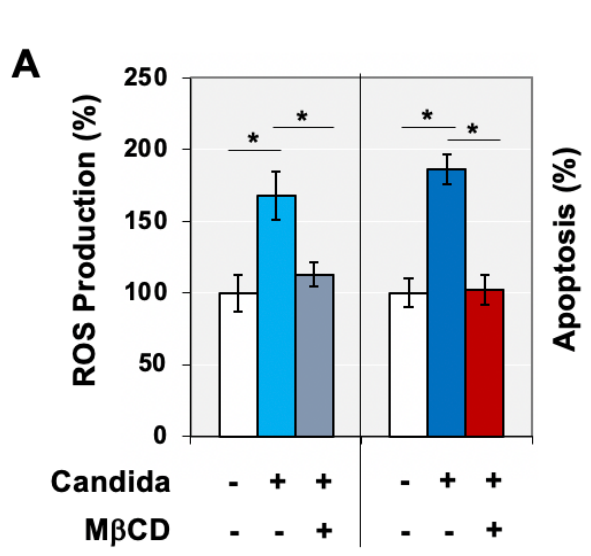

B

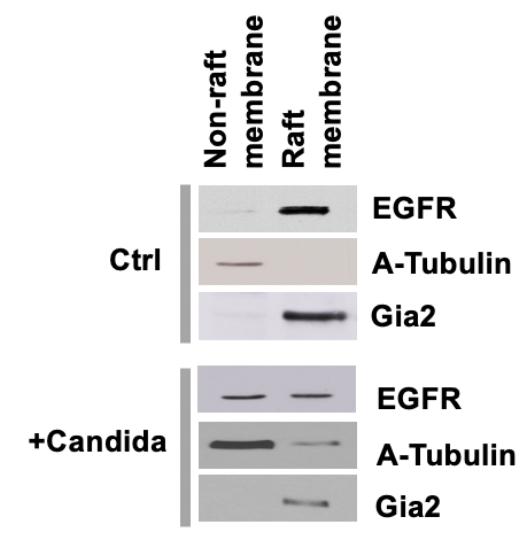

C

D
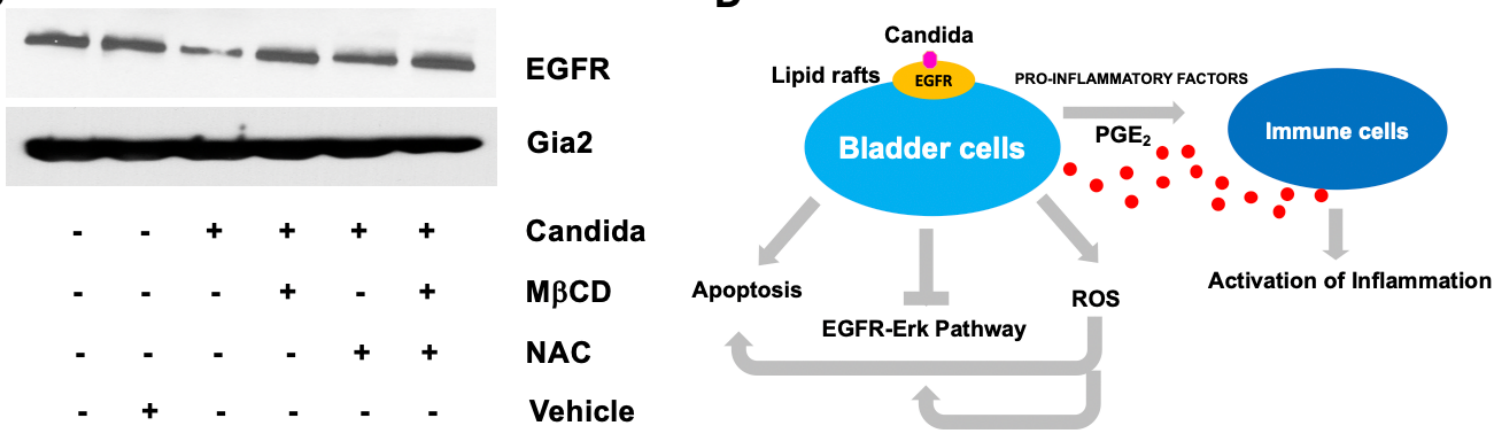


\section{SUPPLEMENTARY FIGURE LEGENDS}

Supplementary Figure 1. Quality Assessment. (A) Distribution of normalized log2 intensities indicated that two samples display different shape of distribution. (B) Scatter plots depict the level of variance among samples.

Supplementary Figure 2. (A-B) The numbers of overlapping upregulated $(\mathbf{A})$ and downregulated (B) genes are shown. 

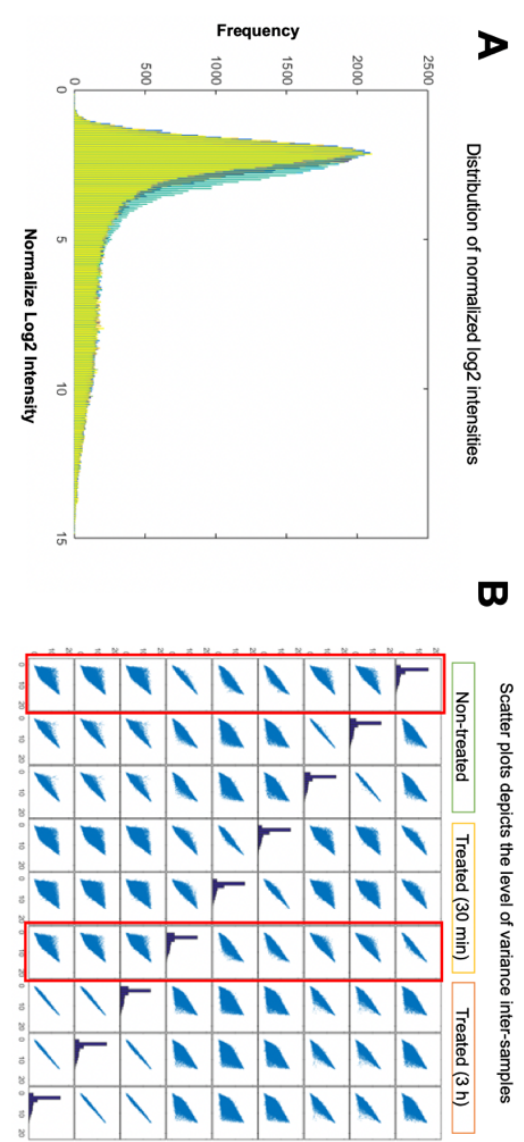

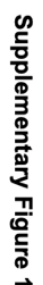




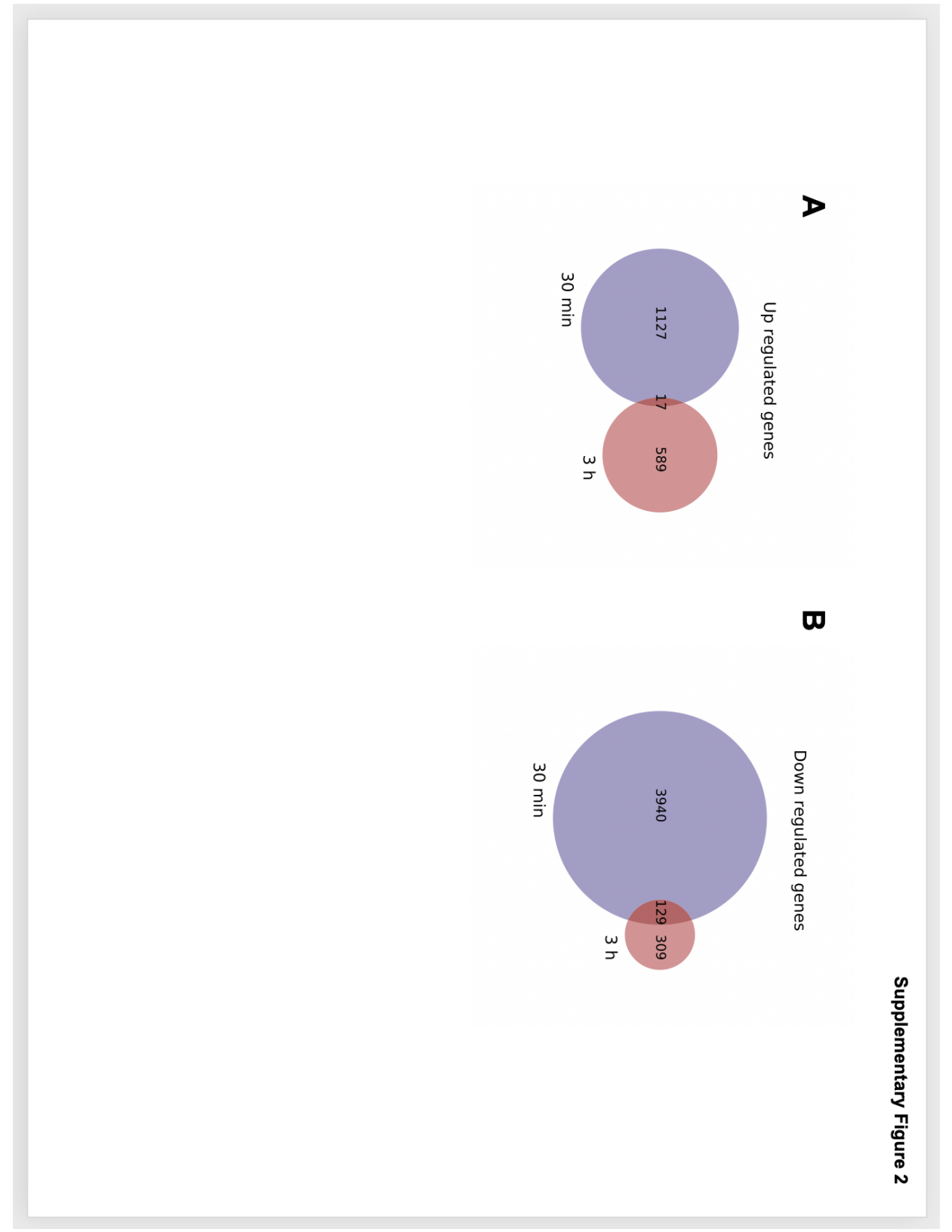




\section{REFFERENCES}

1. Coyne KS, Sexton CC, Thompson CL, et al. (2009) The prevalence of lower urinary tract symptoms (LUTS) in the USA, the UK and Sweden: results from the Epidemiology of LUTS (EpiLUTS) study. BJU Int 104:352-360.

2. Berry SH, Elliott MN, Suttorp M, et al. (2011) Prevalence of symptoms of bladder pain syndrome/interstitial cystitis among adult females in the United States. J Urol 186:540-544.

3. Nickel JC, Stephens A, Landis JR, et al. (2016) Assessment of the Lower Urinary Tract Microbiota during Symptom Flare in Women with Urologic Chronic Pelvic Pain Syndrome: A MAPP Network Study. J Urol 195:356-362.

4. Nickel JC, Stephens A, Landis JR, et al. (2020) Urinary fungi associated with urinary symptom severity among women with interstitial cystitis/bladder pain syndrome (IC/BPS). World J Urol 38:433-446.

5. Jiang HY, Pan LY, Zhang X, Zhang Z, Zhou YY, Ruan B. (2020) Altered gut bacterialfungal interkingdom networks in patients with current depressive episode. Brain Behav 10:e01677.

6. Hof H. (2017) [Fungi in the gut - the gut mycobiome]. Z Gastroenterol 55:772-778.

7. Gu Y, Zhou G, Qin X, Huang S, Wang B, Cao H. (2019) The Potential Role of Gut Mycobiome in Irritable Bowel Syndrome. Front Microbiol 10:1894.

8. Nash AK, Auchtung TA, Wong MC, et al. (2017) The gut mycobiome of the Human Microbiome Project healthy cohort. Microbiome 5:153.

9. Chin VK, Yong VC, Chong PP, Amin Nordin S, Basir R, Abdullah M. (2020) Mycobiome in the Gut: A Multiperspective Review. Mediators Inflamm 2020:9560684.

10. Nagpal R, Neth BJ, Wang S, Mishra SP, Craft S, Yadav H. (2020) Gut mycobiome and its interaction with diet, gut bacteria and alzheimer's disease markers in subjects with mild cognitive impairment: A pilot study. EBioMedicine 59:102950.

11. Gao Z, Guo B, Gao R, Zhu Q, Qin H. (2015) Microbiota disbiosis is associated with colorectal cancer. Front Microbiol 6:20.

12. Escobedo G, Lopez-Ortiz E, Torres-Castro I. (2014) Gut microbiota as a key player in triggering obesity, systemic inflammation and insulin resistance. Revista de investigacion clinica; organo del Hospital de Enfermedades de la Nutricion 66:450-459.

13. Sam Ma Z, Guan Q, Ye C, Zhang C, Foster JA, Forney LJ. (2015) Network analysis suggests a potentially 'evil' alliance of opportunistic pathogens inhibited by a cooperative network in human milk bacterial communities. Scientific reports 5:8275.

14. Carding S, Verbeke K, Vipond DT, Corfe BM, Owen LJ. (2015) Dysbiosis of the gut microbiota in disease. Microbial ecology in health and disease 26:26191.

15. Keay SK, Warren JW. (2002) Is interstitial cystitis an infectious disease? Int J Antimicrob Agents 19:480-483.

16. Duncan JL, Schaeffer AJ. (1997) Do infectious agents cause interstitial cystitis? Urology 49:48-51.

17. Behzadi P, Behzadi E, Ranjbar R. (2015) Urinary tract infections and Candida albicans. Cent European J Urol 68:96-101.

18. Helbig S, Achkar JM, Jain N, et al. (2013) Diagnosis and inflammatory response of patients with candiduria. Mycoses 56:61-69.

19. Badiee P. (2013) Evaluation of human body fluids for the diagnosis of fungal infections. Biomed Res Int 2013:698325.

20. Bukhary ZA. (2008) Candiduria: a review of clinical significance and management. Saudi J Kidney Dis Transpl 19:350-360.

21. Malani AN, Kauffman CA. (2007) Candida urinary tract infections: treatment options. Expert Rev Anti Infect Ther 5:277-284.

22. Birder L, Andersson KE. (2013) Urothelial signaling. Physiol Rev 93:653-680. 
23. Kim J, Ji M, DiDonato JA, et al. (2011) An hTERT-immortalized human urothelial cell line that responds to anti-proliferative factor. In vitro cellular \& developmental biology Animal 47:2-9.

24. Kim J, Keay SK, You S, Loda M, Freeman MR. (2012) A synthetic form of frizzled 8associated antiproliferative factor enhances p53 stability through USP2a and MDM2. PLoS One 7:e50392.

25. Yang W, Chung YG, Kim Y, et al. (2011) Quantitative proteomics identifies a beta-catenin network as an element of the signaling response to Frizzled-8 protein-related antiproliferative factor. Mol Cell Proteomics 10:M110 007492.

26. Kim J, Kim WJ, Liu Z, Loda M, Freeman MR. (2012) The ubiquitin-specific protease USP2a enhances tumor progression by targeting cyclin A1 in bladder cancer. Cell Cycle 11:11231130.

27. Shahid M, Kim M, Yeon A, Andres AM, You S, Kim J. (2018) Quantitative Proteomic Analysis Reveals Caffeine-Perturbed Proteomic Profiles in Normal Bladder Epithelial Cells. Proteomics 18:e1800190.

28. Shahid M, Gull N, Yeon A, et al. (2018) Alpha-oxoglutarate inhibits the proliferation of immortalized normal bladder epithelial cells via an epigenetic switch involving ARID1A. Scientific reports 8:4505.

29. Choi DY, You S, Jung JH, et al. (2014) Extracellular vesicles shed from gefitinib-resistant nonsmall cell lung cancer regulate the tumor microenvironment. Proteomics 14:1845-1856.

30. Zhuang L, Kim J, Adam RM, Solomon KR, Freeman MR. (2005) Cholesterol targeting alters lipid raft composition and cell survival in prostate cancer cells and xenografts. J Clin Invest 115:959-968.

31. McGregor GH, Campbell AD, Fey SK, et al. (2020) Targeting the Metabolic Response to Statin-Mediated Oxidative Stress Produces a Synergistic Antitumor Response. Cancer Res 80:175-188.

32. Li X, Gu J, Zhang Y, et al. (2019) I-arginine alleviates doxorubicin-induced endotheliumdependent dysfunction by promoting nitric oxide generation and inhibiting apoptosis. Toxicology 423:105-111.

33. Solomon KR, Mallory MA, Finberg RW. (1998) Determination of the non-ionic detergent insolubility and phosphoprotein associations of glycosylphosphatidylinositol-anchored proteins expressed on T cells. Biochem J 334 ( Pt 2):325-333.

34. Wang SH, Wang SC, Chen PC, Wang ST, Liu YW. (2017) Induction of cyclooxygenase-2 gene by Candida albicans through EGFR, ERK, and p38 pathways in human urinary epithelium. Med Mycol 55:314-322.

35. Nickel JC, Stephens-Shields AJ, Landis JR, et al. (2019) A Culture-Independent Analysis of the Microbiota of Female Interstitial Cystitis/Bladder Pain Syndrome Participants in the MAPP Research Network. J Clin Med 8.

36. Nickel JC, Stephens A, Landis JR, et al. (2015) Search for Microorganisms in Men with Urologic Chronic Pelvic Pain Syndrome: A Culture-Independent Analysis in the MAPP Research Network. J Urol 194:127-135.

37. Ackerman AL, Anger JT, Khalique MU, et al. (2019) Optimization of DNA extraction from human urinary samples for mycobiome community profiling. PLoS One 14:e0210306.

38. de Turris V, Teloni R, Chiani P, et al. (2015) Candida albicans Targets a Lipid Raft/Dectin1 Platform to Enter Human Monocytes and Induce Antigen Specific T Cell Responses. PLoS One 10:e0142531. 